\title{
Photoelectrochemical Screening of Solar Cell Absorber Layers: Electron Transfer Kinetics and Surface Stabilization SUPPORTING INFORMATION
}

\author{
Diego Colombara ${ }^{a}$, Phillip Dale ${ }^{\mathrm{a}}$, Gabriela Kissling ${ }^{\mathrm{b}}$ Laurence Peter ${ }^{\mathrm{b}, *}$ and Sara Tombolato ${ }^{\mathrm{a}}$ \\ a. Physics and Materials Science Research Unit, Université du Luxembourg, 41 rue du Brill, L-4422 \\ Belvaux, Luxembourg \\ b. Department of Chemistry, University of Bath, Bath BA2 7AY, United Kingdom
}

\section{CdS deposition}

The vertically hanging absorber layers were placed in a stirred solution of $2 \mathrm{mM} \mathrm{CdSO}_{4}$ and $1.5 \mathrm{M}$ $\mathrm{NH}_{4} \mathrm{OH}$ contained in a water jacketed beaker heated to $67^{\circ} \mathrm{C}$. After three minutes thiourea was added to give a solution concentration of $50 \mathrm{mM}$. The samples were removed after eight minutes.

\section{XPS data}

XPS analysis of $\mathrm{K}_{4} \mathrm{Fe}(\mathrm{CN})_{6}$ and $\mathrm{K}_{3} \mathrm{Fe}(\mathrm{CN})_{6}$ powders, purchased from Sigma Aldrich, were performed in order to have a reference position for Fe in the (II) and (III) forms coordinated by $\mathrm{CN}^{-}$groups. These spectra are reported in Figure S1.

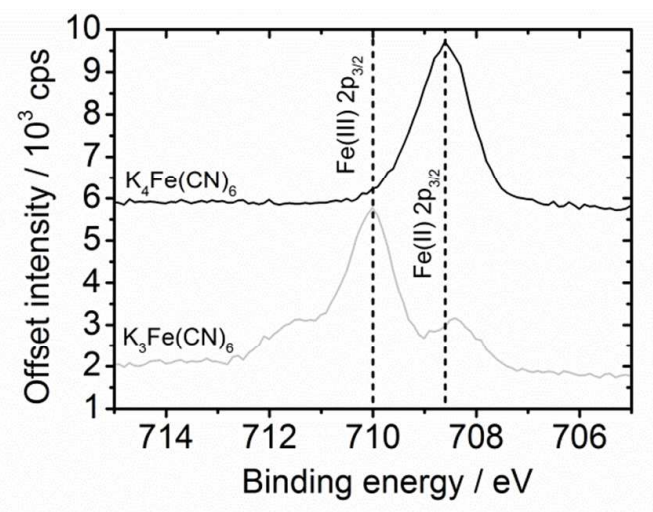

Figure S1. High resolution XPS analysis of $\mathrm{K}_{4} \mathrm{Fe}(\mathrm{CN})_{6}$ and $\mathrm{K}_{3} \mathrm{Fe}(\mathrm{CN})_{6}$ solid powders (Sigma Aldrich).

The In and Ga XPS spectra of the CIGSe films before and after PEC measurements with $0.2 \mathrm{M}$ $\mathrm{K}_{4} \mathrm{Fe}(\mathrm{CN})_{6}+0.2 \mathrm{M} \mathrm{K}_{3} \mathrm{Fe}(\mathrm{CN})_{6}$ are shown in Figure S2. The objective was to try to obtain some information about potentially-occurring atomic dislocations and substitutions. There seems to be a slight shift of the Ga $2 p$ peak to lower binding energies after the PEC measurement, as well as an overall decrease of the intensities. The position of the In peaks remain nearly unchanged. Based on these results, it is difficult to provide a clear conclusion on atomic dislocations or substitutions. 

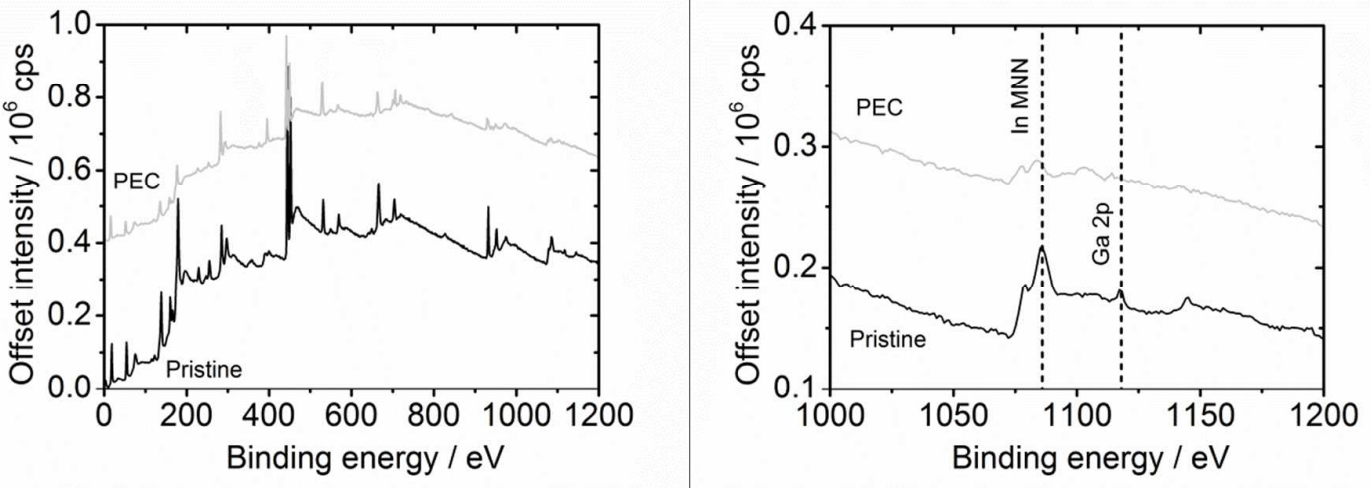

Figure S2. Survey XPS spectra of CIGSe films in pristine state and after PEC measurement with 0.2 $\mathrm{M} \mathrm{K}_{4} \mathrm{Fe}(\mathrm{CN})_{6}+0.2 \mathrm{M} \mathrm{K}_{3} \mathrm{Fe}(\mathrm{CN})_{6}$. Right spectra are a zoom centred around In and Ga peaks.

\section{Kinetics of minority carrier reactions: relating $\boldsymbol{k}_{\text {tr }}$ to $\boldsymbol{k}_{\mathbf{2} \text {,het }}$}

In the case of a 1-eletron redox system, the kinetics of a light-driven process at an illuminated p-type semiconductor electrode in the absence of surface recombination can be described by the general expression

$$
j=q k_{2, \text { het }} n^{\mathrm{a}} O^{\mathrm{b}}
$$

Here $j$ is the current density, $k_{2 \text {,het }}$ is the rate constant for the electron (hole) transfer process, $n$ is the electron concentration $\left(\mathrm{cm}^{-3}\right)$ at the surface of the electrode and $O$ is the concentration of electron acceptor species in solution $\left(\mathrm{cm}^{-3}\right), a$ and $b$ are the reaction orders with respect to electrons and redox acceptor species respectively. If the reaction orders a and $\mathrm{b}$ are both unity and the concentrations $n$ and $O$ are expressed in $\mathrm{cm}^{-3}$, the rate constant $k_{2 \text {,het }}$ in eqn. $\mathrm{S} 1$ has units $\mathrm{cm}^{4} \mathrm{~s}^{-1}$.

For simple outer sphere redox reactions, $k_{2 \text {,het }}$ is given by ${ }^{1}$

$$
k_{2, \text { het }}=v_{\mathrm{n}} \kappa_{\mathrm{n}} \kappa_{\mathrm{el}}
$$

Here $v_{\mathrm{n}}$ is a vibrational frequency along the reaction coordinate (ca. $10^{13} \mathrm{~s}^{-1}$ ) and $\kappa_{\mathrm{n}}, \kappa_{\mathrm{el}}$ are nuclear and electronic coupling terms respectively. The nuclear term depends of the driving force $\Delta G^{0}$ for the electron transfer reaction, and on the reorganization energy, $\lambda$.

$$
\kappa_{\mathrm{n}}=\exp -\left[\frac{\left(\Delta G^{0}+\lambda\right)^{2}}{4 \lambda k_{B} T}\right]
$$

The electronic coupling term $\kappa_{\mathrm{el}}$ is estimated to be of the order of $10^{-22} \mathrm{~cm}^{4}$ for a typical semiconductor/electrolyte system. The maximum value for $\kappa_{n}$ is obtained when $\Delta G^{0}=-\lambda$. This corresponds to a maximum value of $k_{2, \text { het }}=10^{-17} \mathrm{~cm}^{4} \mathrm{~s}^{-1}$.

A useful approximation involves formulating the rate of minority carrier reactions in terms of the surface concentration $n_{\text {surf }}\left(\mathrm{cm}^{-2}\right)$ of minority carriers rather than the volume concentration $\left(\mathrm{cm}^{-3}\right)$ at the surface. The reaction order is reduced by considering that the concentration of solution species $\mathrm{O}$ is constant. So, for an p-type semiconductor under illumination.

$$
j=q k^{\prime} n_{\text {surf }} O=q k_{\text {tr }} n_{\text {surf }}
$$

where $n_{\text {surf }}=n \delta_{s c}, \delta_{s c}$ is a reaction length and $k_{\text {tr }}=k O$. The pseudo-first order rate constant $k_{\text {tr }}$ (with units $\mathrm{s}^{-1}$ ) is related to the rate constant $k_{2 \text {,het }}$ by

$$
k_{\mathrm{tr}}=\frac{k_{2, \text { het }}}{\delta_{\mathrm{sc}}} O
$$


If we assume a concentration of redox species equal to $0.2 \mathrm{M}\left(\equiv 1.2 \times 10^{20} \mathrm{~cm}^{-3}\right)$ and a reaction length of $10 \mathrm{~nm}$, the upper limit of $k_{2 \text {,het }}=10^{-17} \mathrm{~cm}^{4} \mathrm{~s}^{-1}$, a rough estimate of the upper limit for $k_{t r}$ is $10^{10} \mathrm{~s}^{-1}$. This is $10^{8}$ times higher than the value observed for the europium electrolyte.

\section{Kinetics of surface recombination}

The rate of surface recombination is given by

$$
v_{\text {rec }}=\sigma_{\mathrm{n}} v_{\text {th }} p_{x=0} n_{\text {surf }}=\sigma v_{\text {th }} N_{\mathrm{A}} \exp -\left[\frac{q \Delta \phi_{\text {sc }}}{k_{\mathrm{B}} T}\right]=k_{\text {rec }} n_{\text {surf }}
$$

where $\sigma_{\mathrm{n}}$ is the capture cross section for holes (majority carriers), $v_{\mathrm{th}}$ is the thermal velocity of holes, $p$ is the bulk hole concentration, $q \Delta \phi_{\mathrm{sc}}$ is the band bending and $n_{\text {surf }}$ is the surface electron concentration given by

$$
n_{\text {surf }}=\frac{\eta I_{0}}{k_{\text {tr }}+k_{\text {rec }}}
$$

Here $\eta I_{0}$ is the flux of eletrons into the surface defined by the Gärtner equation (cf. eqn. 1 of main text). The recombination rate constant is therefore first order in hole concentration at the surface $(x=0)$.

$$
k_{\text {rec }}=\sigma_{\mathrm{n}} v_{\mathrm{th}} N_{A} \exp -\left[\frac{\Delta \phi_{\mathrm{sc}}}{k_{\mathrm{B}} T}\right]
$$

\section{IMPS Theory}

Intensity-modulated photocurrent spectroscopy (IMPS) $)^{2-7}$ is a convenient way of measuring the rate constants for charge transfer and recombination. The method involves using a small sinusoidal modulation of light intensity (typically less than $10 \%$ of the dc illumination level) and measuring the phase and magnitude of the photocurrent response as a function of frequency. The result, which is usually plotted in the complex plane, can be analyzed to find obtain $k_{\text {tr }}$ and $k_{\text {rec. }}$. The relaxation time constant $\left(k_{\mathrm{tr}}+k_{\mathrm{rec}}\right)^{-1}$ seen in the exponential decay and overshoot of photocurrent transients gives rise to a semi-circular response that is described by the normalized transfer function

$$
\frac{j_{\text {photo }}(\omega)}{q J_{n}}=\frac{k_{t r}+i \omega}{k_{t r}+k_{r e c}+i \omega}
$$

which has real an imaginary parts

$$
\begin{aligned}
& \operatorname{Re}\left[\frac{j_{\text {photo }}(\omega)}{q J_{n}}\right]=\frac{k_{t r}\left(k_{t r}+k_{\text {rec }}\right)+\omega^{2}}{\left(k_{t r}+k_{\text {rec }}\right)+\omega^{2}} \\
& \operatorname{Im}\left[\frac{j_{\text {photo }}(\omega)}{q J_{n}}\right]=\frac{k_{\text {rec }} \omega}{\left(k_{t r}+k_{\text {rec }}\right)+\omega^{2}}
\end{aligned}
$$

Here $J_{n}$ is the modulated flux of electrons into the surface. The IMPS response corresponds to a semicircle with a high frequency (HF) intercept on the real axis at 1 and a low frequency intercept equal to $k_{t r} /\left(k_{t r}+k_{r e c}\right)$. he maximum of the semicircle occurs at a radial frequency $\omega_{\max }=k_{t r}+k_{\text {rec }}$. Comparison with the transient response described in the preceding section shows that the HF limit corresponds to the instantaneous photocurrent and the LF limit to the steady state photocurrent. The time constant of the transient response is $1 / \omega_{\max }$. 


\section{Experiments using p-GaAs}

p-type $10^{16} \mathrm{~cm}^{-3} \mathrm{Zn}$-doped GaAs wafers purchased from Wafer Technology Ltd. were cleaved to a size of approximately $2 \mathrm{~cm}^{2}$, attached to a soda lime glass substrate and contacted with silver epoxy. A CdS layer was deposited under the same conditions as those used to coat CIGSe samples. Figure S3 compares the chopped photocurrent responses of bare and CdS-coated p-GaAs in the hexacyanoferrate electrolyte. The similarity to the results obtained with CIGSe suggests that the CdScoating method is more widely applicable.

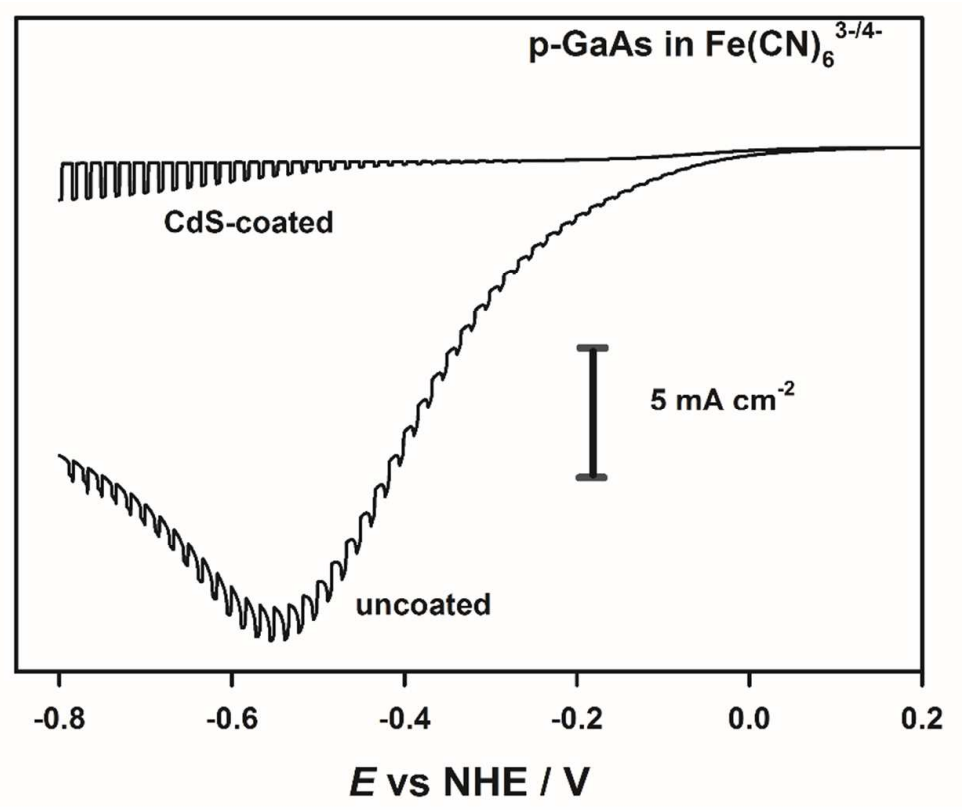

Figure S3. Photocurrent response of p-GaAs electrodes in hexacyanoferrate electrolyte illustrating stabilization by deposition of a thin CdS layer (compare with Figures 1 and 4 of main text, which show responses for $\mathrm{p}$-CIGSe).

\section{References}

1. Pomykal, K. E.; Fajardo, A. M. ; Lewis, N. S., J. Phys. Chem., 1996, 100, 3652-3664.

2. Ponomarev, E. A.; Peter, L. M., J. Electroanal. Chem., 1995, 396, 219-226.

3. Peter, L. M. Chem. Rev., 1990, 90, 753-769.

4. Li, J.; Peter, L. M., J. Electroanal. Chem.., 1985, 193, 27-47.

5. Li, J.; Peter, L. M., J. Electroanal. Chem., 1986, 199, 1-26.

6. Peter, L. M., Chem. Rev. 1990, 90, 753-769.

7. Peter, L. M.; Vanmaekelbergh D., in Adv. Electrochem. Sci. Eng., eds. R.C. Alkire, D.M. Kolb, Weinheim, 1999, vol. 6, pp. 77-163. 